\title{
Psychische Störungen bei Patienten mit muskuloskelettalen und kardiovaskulären Erkrankungen im Vergleich zur Allgemeinbevölkerung
}

\author{
Harald Baumeister ${ }^{1}$, Michael Höfler ${ }^{2}$, Frank Jacobi ${ }^{3}$, Hans-Ulrich Wittchen ${ }^{3}$, \\ Jürgen Bengel ${ }^{4}$ und Martin Härter ${ }^{1}$
}

${ }^{1}$ Abteilung für Psyhiatrie und Psychotherapie, Universitätsklinikum Freiburg

${ }^{2}$ Max-Planck-Institut für Psychiatrie München

${ }^{3}$ Abteilung für klinishe Psychologie, Universität Dresden

${ }^{4}$ Abteilung für Rehabilitationspsychologie, Universität Freiburg

\begin{abstract}
Zusammenfassung. Hintergrund: Ein signifikanter Anteil der Patienten mit einer chronischen körperlichen Erkrankung weist eine komorbide psychische Störung auf. Ob und in welchem Ausmaß sich die Prävalenzraten psychischer Störungen bei Patienten mit einer chronischen Erkrankung von denen der Allgemeinbevölkerung unterscheiden, ist bislang noch kaum untersucht. Fragestellung: Die vorliegende epidemiologische Studie untersucht geschlechts- und altersadjustierte 4-Wochen, 12-Monats- und Lebenszeitprävalenzen psychischer Störungen bei Rehabilitationspatienten mit muskuloskelettalen und kardiovaskulären Erkrankungen im Vergleich zu Prävalenzraten der Allgemeinbevölkerung. Methode: Die Daten der drei Stichproben $(N=4192)$ basieren jeweils auf einem zweistufigen, epidemiologischen Untersuchungsansatz mit einer schriftlichen Befragung der Patienten bzw. Probanden zu ihrem psychischen Befinden (GHQ-12; M-CIDI-S) und einem anschließenden Interview (M-CIDI) bei einem randomisiert ausgewählten Teil der Gesamtstichprobe. Ergebnisse: Mit adjustierten Lebenszeitprävalenzen von 59.3\% (OR: 1.6) und 56.2\% (OR: 1.4) weisen die Patienten mit einer muskuloskelettalen und kardiovaskulären Erkrankung im Vergleich zur Allgemeinbevölkerung (47.9\%) eine deutlich erhöhte Prävalenz psychischer Störungen auf. Am häufigsten sind affektive Störungen ( $22.5 \%$ bis $34.9 \%)$ und Angststörungen (18.4\% bis 33.8\%). Schlussfolgerung: Der im Vergleich zur Allgemeinbevölkerung deutliche Zusammenhang zwischen chronischen körperlichen Erkrankungen und psychischen Störungen verdeutlicht die Bedeutsamkeit einer verstärkten Diagnostik und Behandlung komorbider psychischer Störungen bei chronisch erkrankten Patienten.

Schlüsselwörter: Psychische Störungen, muskuloskelettale Krankheiten, kardiovaskuläre Krankheiten, Komorbidität, Epidemiologie, chronische Erkrankungen
\end{abstract}

Mental disorders in patients with muscoskeletal and cardiovascular diseases in comparison to the general population

Abstract. Background: A significant part of patients with chronic diseases have comorbid mental disorders. However, by now it is nearly unexplored if and to what extend the prevalence rates of mental disorders in patients with chronic diseases differ from the rates of the general population. Objective: The present epidemiologic study investigates sex- and age-adjusted 4-week, 12-months, and lifetime prevalence rates of mental disorders in inpatients with musculoskeletal and cardiovascular diseases compared to prevalence rates of the general population. Methods: In each sample $(N=4192)$, the data based on a two-stage epidemiologic design. The first stage entailed the use of a screening questionnaire for mental disorders (GHQ-12; M-CIDI-S). The second stage consisted of an interview (M-CIDI) of a randomised part of the sample. Results: The adjusted lifetime prevalence in both clinical samples (musculoskeletal: $59.3 \%$, OR 1.6; cardiovaskular: $56.2 \%$, OR 1.4 ) is high compared to the rate of the general population (47.9\%). Affective disorders $(22.5 \%$ to $34.9 \%)$ and anxiety disorders $(18.4 \%$ to $33.8 \%)$ are the most common disorders. Conclusions: Compared to the general population there is a clear correlation between chronic diseases and mental disorders, that shows the importance of an improved diagnostic and treatment of patients suffering from comorbid mental disorders.

Keywords: mental disorders, musculoskeletal diseases, cardiovascular diseases, comorbidity, epidemiology, chronic diseases

Über eine enge Beziehung zwischen somatischen und psychischen, insbesondere depressiven Beschwerden und Störungen ist konsistent in vielen Studien berichtet worden (Penninx et al., 2001; Pratt et al., 1996; Wells, Golding \& Burnam, 1988). Die Häufigkeit depressiver
Störungen bei Patienten mit somatischen Erkrankungen wird in einer Zusammenfassung der 1960 bis 1990 durchgeführten Studien, alle auf der Basis klinischer semistrukturierter oder standardisierter psychiatrischer Interviews, auf $0 \%$ bis $54 \%$ und von $2 \%$ bis $15 \%$ für eine 
Major Depression geschätzt (Cohen-Cole, Brown \& McDaniel, 1993). Werden Studien betrachtet, die für die Schätzung von Prävalenzraten Selbstbeurteilungs- und Screeningverfahren eingesetzt haben, schwanken die Raten für depressive Symptome noch stärker $(2 \%$ bis $70 \%$, Cohen-Cole et al., 1993).

Die breite Variation von Prävalenzraten für psychische Störungen ist auch aus den Studien von Patienten mit muskuloskelettalen (z. B. Frank et al., 1988; Härter, Reuter, Weisser et al., 2002; Magni, Marchetti, Moreschi, Merskey \& Lucchini, 1993; Polatin, Kinney, Gatchel, Lillo \& Mayer, 1993) und kardiovaskulären Erkrankungen bekannt (z. B. Chignon, Lepine \& Ades, 1993; Frasure-Smith, Lespérance \& Talajic, 1993; Gonzalez et al., 1996; Härter \& Bengel, 2001). Keine der Studien kann allerdings eine Aussage darüber treffen, ob die unterschiedlichen Prävalenzraten auf die höhere Belastung durch eine somatische Erkrankung zurückzuführen sind und ob sich diese Raten in signifikanter Weise von Raten psychischer Störungen einer gesunden Kontrollgruppe bzw. der Allgemeinbevölkerung unterscheiden.

Im Gegensatz zu den umfangreichen, methodisch hochwertigen epidemiologischen Studien zur Prävalenz psychischer Störungen in der Allgemeinbevölkerung (Kessler et al. 1994; Robins \& Regier, 1991; Wittchen, 2000) basieren die Prävalenzschätzungen für komorbide psychische Störungen beim Vorliegen einer chronischen somatischen Erkrankung auf klinischen (selektiven) Stichproben und einem den jeweiligen Fragestellungen angepassten Studiendesign mit verschiedenen Messinstrumenten und unterschiedlichen Prävalenzzeitfenstern (z. B. 6 Monate, 12 Monate, Lebenszeit). Epidemiologische Studien gibt es in diesem Bereich bis auf die NIMH Epidemiologic Catchment Area (ECA) Studie (Wells et al., 1988, 1989) und eigene Untersuchungen (Härter \& Bengel, 2001; Härter, Reuter, Aschenbrenner et al., 2001; Härter, Reuter, Weisser et al., 2002) bislang nicht.

Ein Vergleich von Prävalenzen psychischer Störungen bei körperlichen Erkrankungen mit Raten aus der Allgemeinbevölkerung bedarf eines methodisch vergleichbaren epidemiologischen Vorgehens mit einer repräsentativen Stichprobe und eines standardisierten Verfahren zur Diagnosestellung. Bislang konnte einzig in der ECA-Studie, bezogen auf eine nicht chronisch kranke Kontrollgruppe (Ausschluss von acht wesentlichen chronischen Erkrankungen), gezeigt werden, dass chronisch somatisch Erkrankte, unter Berücksichtigung von Alters- und Geschlechtseffekten, höhere Prävalenzraten psychischer Störungen aufweisen (Wells et al., 1988). Für die untersuchten Personen mit muskuloskelettalen Erkrankungen (Arthritiden) ergaben sich Prävalenzraten von 50\% (Lebenszeit) bzw. 25\% (6 Monate) und für Patienten mit einer kardiovaskulären Erkrankung Prävalenzen von 34\% bzw. $22 \%$ bei Hypertonie, sowie von $50 \%$ bzw. $35 \%$ bei koronarer Herzerkrankung. Die Prävalenzraten für die Gruppe ohne chronische somatische Erkrankungen liegen bei 33\% (Lebenszeit) und 18\% (6 Monate). Eingeschränkt werden die Ergebnisse allerdings, da die ECAStudie noch auf den Kriterien des DSM-III und dem Diag- nostic Interview Schedule (DIS) basierte und nur auf die drei psychischen Kategorien „Affektive Störungen“, „Angststörungen“ und „Substanzabhängigkeit/-missbrauch" fokussierte. Außerdem beruhten die Angaben zu den chronischen Erkrankungen nur auf Selbstauskünften der Probanden (keine ärztlich gesicherten Diagnosen).

In eigenen epidemiologischen Untersuchungen konnten an repräsentativen Stichproben von Rehabilitationspatienten mit muskuloskelettalen und kardiovaskulären Erkrankungen mittels des auf DSM-IV-Kriterien basierenden Munich-Composite International Diagnostic Interview (M-CIDI) Prävalenzraten komorbider psychischer Störungen erhoben werden (Härter \& Bengel, 2001; Härter, Reuter, Weisser et al., 2002). Für die Patienten mit einer muskuloskelettalen Erkrankung ergaben sich Prävalenzraten von $65 \%$ (Lebenszeit), 47\% (12 Monate) und $31 \%$ (4 Wochen) und für die Patienten mit einer kardiovaskulären Erkrankung Prävalenzen von 55\%, 34\% und $20 \%$ (Härter \& Bengel, 2001). Im Gegensatz zur ECAStudie (Wells et al., 1988) beruhen die Prävalenzraten unserer Untersuchung auf einer Stichprobe von Patienten mit ärztlich gesicherten somatischen Diagnosen.

Bislang ließ sich aber nur approximativ schätzen, ob und in welchem Ausmaß die vorgefundenen psychischen Störungen in den verschiedenen Erkrankungsgruppen im Vergleich zu den Prävalenzraten aus repräsentativen Bevölkerungserhebungen in Deutschland bzw. international (Kessler et al., 1994; Wittchen, 2000) erhöht sind, da Moderatorvariablen wie Alter und Geschlecht der Probanden, aufgrund unterschiedlicher Ausprägungen in den Stichproben, die Vergleichbarkeit der Prävalenzraten einschränken.

Ziel des Beitrags ist, erstmals geschlechts- und altersadjustierte Prävalenzraten psychischer Störungen für chronisch somatisch erkrankte Patienten mit ärztlich gesicherten Diagnosen im Vergleich zur Allgemeinbevölkerung zu analysieren. Aufgrund der Arbeiten von Wells et al. (1988, 1989) und unseren eigenen Ergebnissen (Härter \& Bengel, 2001; Härter, Reuter, Weisser et al., 2002) ist zu erwarten, dass Patienten mit chronischen Erkrankungen im Vergleich zur Allgemeinbevölkerung auch nach Alters- und Geschlechtsadjustierung signifikant höhere Prävalenzraten psychischer Störungen aufweisen.

\section{Methodik}

\section{Studiendesign}

Die Daten der Patienten mit einer muskuloskelettalen und kardiovaskulären Erkrankung wurden im Rahmen des Forschungsprojektes „Epidemiologie psychischer Störungen in der medizinischen Rehabilitation" erhoben, welches von 1998 bis 2001 durchgeführt wurde (Härter \& Bengel, 2001). Als Vergleichsgruppe dienen die Daten einer repräsentativen Stichprobe zu psychischen Störungen in der Allgemeinbevölkerung (Bundesgesundheitssurvey 1998/99, Zusatzsurvey „Psychische Störungen“; Wittchen, 2000). 
Die epidemiologischen Studiendesigns der beiden Untersuchungen weisen neben wesentlichen Überschneidungen auch einige Unterschiede auf. Da zu beiden Vorgehensweisen ausführliche Methodenbeschreibungen vorliegen (Härter \& Bengel, 2001; Härter, Reuter, Weisser et al., 2002; Jacobi et al., 2002), soll in diesem Beitrag nur auf die wesentlichen Gemeinsamkeiten und Unterschiede eingegangen werden.

In der Studie Epidemiologie psychischer Störungen in der medizinischen Rehabilitation wurden mit standardisierten klinischen Interviews die Häufigkeit psychischer Störungen bei Patienten mit muskuloskelettalen und kardiovaskulären Erkrankungen untersucht. Die Stichproben beider Indikationen stammen aus zehn Rehabilitationskliniken in Süd-West-Deutschland. Nach Durchführung eines Screenings mit dem General Health Questionnaire in der 12-Item-Version (GHQ-12; Goldberg \& Williams, 1988 ) wurden schwerpunktmäßig bei denjenigen Patienten klinische Interviews durchgeführt, bei denen aufgrund der Ergebnisse der Screeninguntersuchung der Verdacht auf das Vorliegen einer psychischen Beeinträchtigung bestand. Auf der Grundlage der einzelnen GHQ-Werte wurden $10 \%$ der Patienten mit niedrigem (0-4), 30\% mit mittlerem (5-7) und 50\% mit hohem GHQ-Wert (8-12) randomisiert zur zweiten Untersuchungsphase, dem $\mathrm{Mu}-$ nich-Composite International Diagnostic Interview, ausgewählt (M-CIDI; Wittchen, Weigel \& Pfister, 1996). Dem Oversampling von Probanden mit höherem GHQWert wird bei der späteren Auswertung durch entsprechende Gewichtung Rechnung getragen.

Die Daten aus der Allgemeinbevölkerung wurden im Rahmen des Zusatzsurveys „Psychische Störungen “ des Bundes-Gesundheitssurveys 1998 erhoben, der ersten bundesweiten epidemiologischen Untersuchung psychischer Gesundheit der erwachsenen Bevölkerung in Deutschland (Bellach, 1999; Jacobi et al., 2002). Der Kernsurvey wurde vom Robert Koch-Institut durchgeführt, der Zusatzsurvey „Psychische Störungen“ ist eines von mehreren Zusatzmodulen des Kernsurveys und wurde vom Max-Planck-Institut für Psychiatrie durchgeführt.

Die Datenerhebung des Zusatzsurveys basierte auf einem zweistufigen Design: Zunächst wurden alle Teilnehmer des Kernsurveys $(N=7124)$ mit dem Münchner Composite International Diagnostic Interview-Stammfra- gebogen (M-CIDI-S) gescreent. Daraufhin wurden alle Screening-positiven und $50 \%$ der Screening-negativen Teilnehmer mit dem M-CIDI untersucht. Teilnehmer des Kernsurveys, die älter als 65 Jahre waren, wurden aus der Studie ausgeschlossen. Die Stichprobengröße des Zusatzsurveys beträgt $N=4181$ (konditionale Response-Rate: $87.6 \%$; Jacobi et al., 2002).

\section{Diagnostik}

Das Munich-Composite International Diagnostic Interview (DIA-X/M-CIDI, Wittchen \& Pfister, 1997) ist eine überarbeitete und computergestützte Version des World Health Organisation-CIDI (WHO 1990, 1997). Es ermöglicht eine reliable und valide Erhebung von Symptomen, Syndromen und Diagnosen psychischer Störungen nach DSM-IV und ICD-10 (4 Wochen, 12 Monats- und Lebenszeitprävalenz). Der Vorteil dieses Verfahrens liegt in der hohen Objektivität und Interraterreliabilität (Wittchen et al., 1991; Wittchen, Weigel \& Pfister, 1996).

Die in der Ergebnisdarstellung berücksichtigten Diagnosen entstammen den Hauptkategorien Substanzbezogene Störungen, Affektive Störungen, Angststörungen, Somatoforme Störungen, Essstörungen und mögliche psychotische Störungen (Tab. 1).

\section{Interviewer}

In allen drei Gruppen wurde das M-CIDI von klinisch erfahrenen Interviewern durchgeführt, die in einem standardisierten zweitägigen Interview-Training geschult wurden. Das Training umfasste eine detaillierte Erarbeitung der einzelnen Sektionen des M-CIDI entsprechend des Instruktionsmanuals zur Durchführung des Interviews (Wittchen \& Pfister, 1997). Während der Datenerhebung fanden fortlaufend Supervisionen statt.

\section{Auswertung}

Der aus der Datenbankarbeit mittels des M-CIDI-Datenbankmoduls (Wittchen \& Pfister, 1997) resultierende Datensatz umfasst sowohl die Interviewdaten der 4181

Tabelle 1. Die den Ergebnissen zugrunde liegenden spezifischen Diagnosen gemäß der ICD-10-Klassifikation

\begin{tabular}{ll}
\hline Diagnosehauptgruppen & enthaltene spezifische Diagnosen \\
\hline Substanzbezogene Störungen $^{1}$ & F10, F11, F12, F13, F14, F15, F16, F17, F18, F19 \\
Affektive Störungen & F30, F31, F32, F33, F34.1, F06.3 \\
Angststörungen & F40.0, F40.1, F40.2, F40.9, F41.0, F41.1, F42.0, F06.4 \\
Somatoforme Störungen & F45.0, F45.1, F45.2, F45.4 \\
Andere Störungen & F2 \\
& F5: F50.0, F50.1, F50.2, F50.3 \\
\hline
\end{tabular}

Anmerkungen: ${ }^{1}$ Die Substanzbezogenen Störungen umfassen je psychotrope Substanz - mit Ausnahme von F17 - die Subtypen F1x.1 Schädlicher Gebrauch und F1x.2 Abhängigkeitssyndrom (Im Fall von F17 nur F1x.2). ${ }^{2}$ Die organisch bedingten Störungen (F06.x) wurden aufgrund der im CIDIInterview nicht ausreichend zu klärenden Ätiologie unter die entsprechenden psychischen Störungen subsumiert. 
Probanden aus der Allgemeinbevölkerung als auch die gesamten Interviews aus den klinischen Stichproben. Das Vorgehen der Datengewinnung und -aufbereitung ist somit bei den klinischen Stichproben und den Daten des Bundesgesundheitssurveys identisch.

Beim Vergleich zwischen den beiden Gruppen von Rehabilitationspatienten und der Allgemeinbevölkerung wurde nach Alter- und Geschlechtseffekten kontrolliert. Hierzu war es notwendig, den Altersrange der klinischen Stichproben und der BGS-Stichprobe anzupassen. Das Alter der Probanden musste aufgrund dessen zwischen 22 (jüngster Patient aus der klinischen Stichprobe) und 65 (ältester Patient aus der BGS-Stichprobe) Jahren liegen.

Die Datenanalyse erfolgte mittels Stata Statistical Software (StataCorp., 1999). Um der Gewichtung und Stratifizierung der Stichproben (nach Alter, Geschlecht, Region, Stichprobenfaktoren) Rechnung zu tragen, wurden die SVY (survey) Kommandos von Stata angewendet, die einen adäquaten Umgangs mit gewichteten und stratifizierten Daten ermöglichen. Neben den Prävalenzraten psychischer Störungen mit 95\%-Konfidenz-Intervallen zu den drei Stichproben wurden Odds Ratios (OR) mit 95\%-Konfidenz-Intervallen berechnet. Die gewichteten Prävalenzraten der klinischen Stichproben wurden durch logistische Regressionen an die Prävalenzraten in der BGS-Stichprobe angeglichen. Sie geben somit wieder, welche Prävalenzen psychischer Störungen man in den Reha-Stichproben erwarten würde, wenn deren Alters- und Geschlechtsverteilung der (gewichteten) Verteilung in der BGS-Stichprobe entsprechen würde.

\section{Stichproben}

Patienten mit muskuloskelettalen Erkrankungen: Insgesamt wurden 811 Patienten mit dem GHQ-12 gescreent, von denen 462 einen niedrigen, 163 einen mittleren und 186 einen hohen GHQ-Wert aufweisen. Von diesen Patienten liegen 194 Interviews vor, die mindestens die Sektionen Angststörungen und Depression umfassen. Zur Datenanalyse wurden von den 194 Interviews 7 wegen des Alters der Patienten ( $>65$ ) von der Analyse ausge- schlossen. Von den verbleibenden 187 Patienten weisen 66 einen niedrigen, 60 einen mittleren und 61 einen hohen GHQ-Wert auf. In der Gruppe der Patienten mit muskuloskelettalen Erkrankungen $(N=187)$ haben $71 \%$ der Patienten eine Dorsopathie, $13 \%$ eine Arthropatie, 5\% Weichteilrheumatismen und $11 \%$ sonstige muskuloskelettale Erkrankungen als Erstindikation. Die soziodemographischen Daten variieren nur unwesentlich zwischen der gewichteten und ungewichteten Stichprobe (Tab. 2).

Patienten mit kardiovaskulären Erkrankungen: Es wurden 787 Patienten mit dem GHQ-12 gescreent, von denen 503 einen niedrigen, 144 einen mittleren und 140 einen hohen GHQ-Wert aufweisen. Zur Datenanalyse mussten von den vorliegenden 164 Interviews 48 wegen des Alters der Patienten ( $>65$ ) von der Analyse ausgeschlossen werden. Von den verbleibenden 116 Patienten weisen 36 einen niedrigen, 33 einen mittleren und 47 einen hohen GHQ-Wert auf. In der Gruppe der Patienten mit kardiovaskulären Erkrankungen $(N=116)$ haben 51\% der Patienten eine koronare Herzerkrankung, $16 \%$ einen Myokardinfarkt, $5 \%$ eine Rhythmusstörung, $5 \%$ weisen eine Hypertonie auf und $23 \%$ haben eine sonstige kardiovaskuläre Erkrankung als Erstindikation. Die Geschlechtsverteilung ist bei der gewichteten Darstellung von $79 \%$ männliche Patienten auf $85 \%$ gestiegen und die Erwerbstätigkeit von $56 \%$ auf $51 \%$ gesunken. Die im Vergleich zu den beiden anderen Stichproben sehr niedrige Erwerbstätigkeitsrate geht auf den deutlich höheren Altersdurchschnitt der Stichprobe kardiovaskulär erkrankter Patienten zurück (Tab. 2).

Allgemeinbevölkerung: Aus der BGS-Stichprobe wurden 292 Interviews wegen des Alters der Probanden $(<22)$ von der Analyse ausgeschlossen, so dass 3889 Teilnehmer in der Stichprobe verblieben.

\section{Ergebnisse}

Die Prävalenzraten irgendeiner psychischen Störung in der BGS-Stichprobe betragen 22.9\% (4 Wochen), 35.2\% (12 Monate) und 47.9\% (Lebenszeit). In der kardiovaskulären Stichprobe ergeben sich Prävalenzen von $22.4 \%$,

Tabelle 2. Soziodemographische Charakteristika (ungewichtet und gewichtet) der Patienten mit muskuloskelettalen (MS) und kardiovaskulären (KV) Erkrankungen, sowie Personen aus der Allgemeinbevölkerung (BGS)

\begin{tabular}{lcccccc}
\hline & \multicolumn{2}{c}{ MS } & \multicolumn{2}{c}{ KV } & BGS \\
& \multicolumn{2}{c}{$N=187^{1}$} & & $N=116^{1}$ & & $N=3889^{1}$ \\
\cline { 2 - 7 } & ungewichtet & gewichtet & ungewichtet & gewichtet & ungewichtet & gewichtet \\
\hline Geschlecht, M : F (\%) & $53: 47$ & $56: 44$ & $79: 21$ & $85: 15$ & $45: 55$ & $48: 52$ \\
Alter, mean (SD) & $47(10)$ & $48(10)$ & $54(7)$ & $55(7)$ & $44(12)$ & $43(12)$ \\
Familienstand (verheiratet, \%) & 67 & 66 & 78 & 82 & 69 & 67 \\
Voll-/Teilzeitbeschäftigt (\%) & 74 & 75 & 56 & 51 & 67 & 68 \\
\hline
\end{tabular}

Anmerkungen: ${ }^{1}$ Aufgrund der Altersanpassung zwischen den Stichproben (nur die 22-65-Jährigen gingen in die Analysen ein) wurden 7 Patienten der MS-Gruppe (ursprünglich $N=194$ ), 48 der KV-Gruppe (ursprünglich $N=164$ ) und 292 der BGS-Gruppe (ursprünglich $N=4181$ ) ausgeschlossen. 


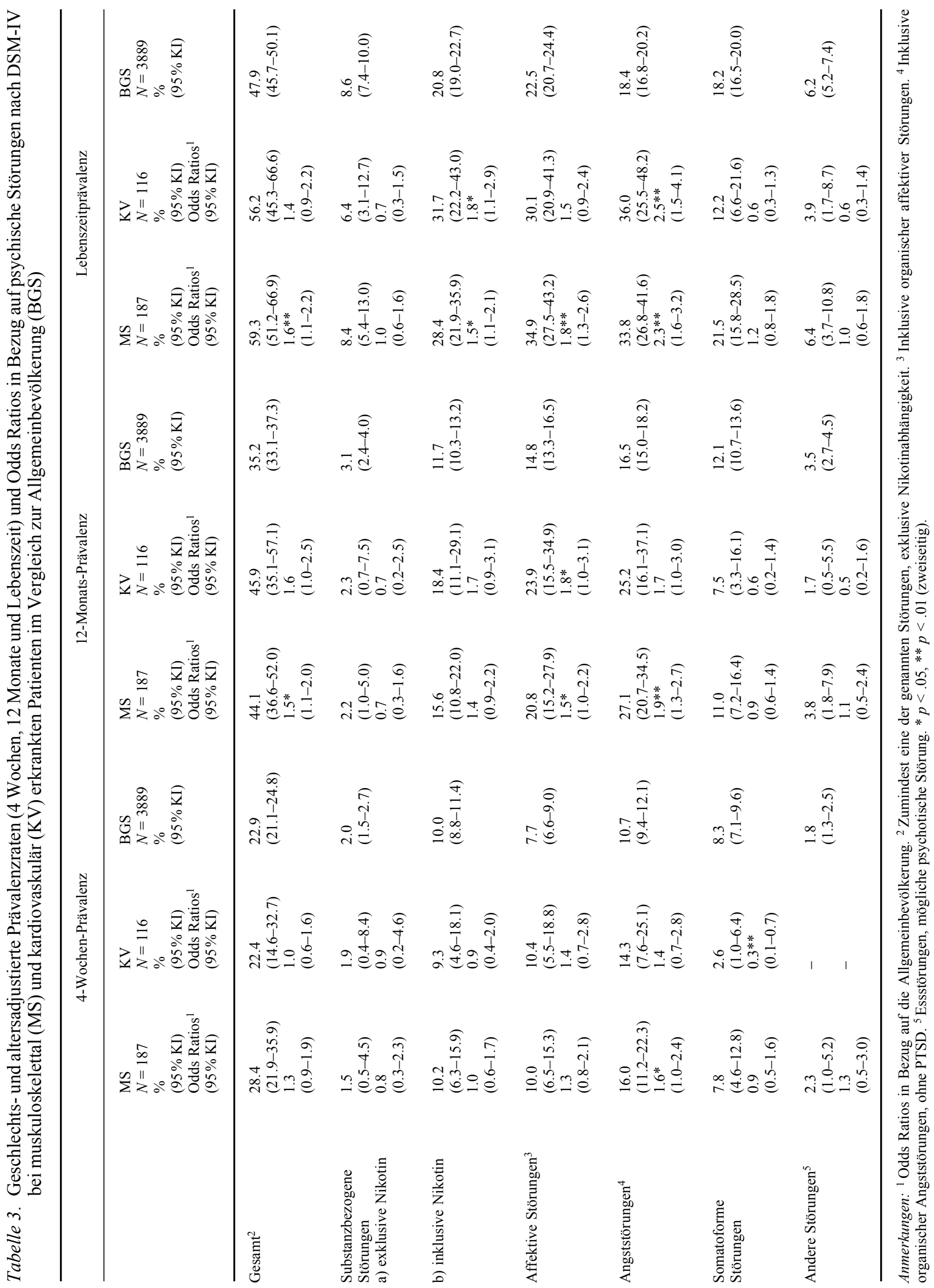


$45.9 \%$ und $56.2 \%$ und in der muskuloskelettalen Stichprobe von $28.4 \%, 44.1 \%$ und $59.3 \%$ (Tab. 3).

Über alle Zeitfenster und Stichproben hinweg sind die häufigsten Diagnosen affektive und Angststörungen. Die Punktprävalenzen (4 Wochen) für affektive und Angststörungen betragen in der BGS-Stichprobe $7.7 \%$ und $10.7 \%$, in der kardiovaskulären Stichprobe $10.4 \%$ und $14.3 \%$ und in der muskuloskelettalen Stichprobe $10.0 \%$ und $16.0 \%$.

Neben den affektiven und den Angststörungen sind die Prävalenzraten somatoformer Störungen in der muskuloskelettalen und der BGS-Stichprobe mit $7.8 \%$ (Punktprävalenz) und $8.3 \%$ noch ähnlich hoch, während die Patienten mit einer kardiovaskulären Erkrankung nur zu 2.6\% somatoforme Störungen aufweisen.

Die Prävalenzraten substanzbezogener Störungen sind maßgeblich davon bestimmt, ob sie mit oder ohne Nikotinabhängigkeit wiedergegeben werden. Die Punktprävalenzen betragen in der muskuloskelettalen Stichprobe $10.2 \%$ (inklusive Nikotin) und $1.5 \%$ (exklusive Nikotin), in der kardiovaskulären Stichprobe $9.3 \%$ und $1.9 \%$ und in der BGS-Stichprobe $10.0 \%$ und $2.0 \%$.

Die Prävalenzraten bezüglich den Essstörungen und den möglichen psychotischen Störungen, die unter, ,andere Störungen" subsumiert wurden, sind in allen drei Stichproben relativ niedrig und weichen zu allen drei Zeitfenstern nicht signifikant voneinander $a b$.

Im Vergleich zwischen der muskuloskelettalen und der BGS-Stichprobe sind die Prävalenzraten in der muskuloskelettalen Stichprobe in allen Diagnosekategorien ähnlich hoch bis deutlich höher als in der BGS-Stichprobe. Über alle drei Zeitfenstern hinweg gleichen sich die Prävalenzraten der beiden Stichproben annähernd in Bezug auf die substanzbezogenen Störungen exklusive Nikotin (OR: 0.8 (4 Wochen), 0.7 (12 Monate), 1.0 (Lebenszeit)), die somatoformen Störungen (OR: 0.9, 0.9, 1.2) und in Bezug auf andere Störungen (OR: 1.3, 1.1, 1.0). Eine deutlich erhöhte Prävalenz findet sich hingegen bei den substanzbezogenen Störungen inklusive Nikotin (OR: 1.0, 1.4, 1.5*), bei den affektiven Störungen (1.3, $1.5^{*}, 1.8^{* *}$ ), sowie bei den Angststörungen (OR: 1.6*, $\left.1.9^{* *}, 2.3^{* *}\right)$. Entsprechend der ähnlich hohen bis deutlich höheren Prävalenz in den einzelnen Diagnosegruppen ergibt sich auch eine deutlich höhere Prävalenz psychischer Störungen insgesamt (OR: 1.3, 1.5* $1.6^{* *}$ ).

Der Vergleich der Prävalenzraten psychischer Störungen von Patienten mit kardiovaskulären Erkrankungen und Probanden aus der BGS-Stichprobe fällt weniger eindeutig aus. Insgesamt ergeben sich Odds Ratios von 1.0 (4 Wochen), 1.6 (12 Monate) und 1.4 (Lebenszeit). Eine durchgehend deutlich niedrigere Prävalenzrate in der kardiovaskulären Stichprobe findet sich bezüglich den somatoformen Störungen (OR: 0.3**, 0.6, 0.6) und den sonstigen Störungen (OR: - , 0.5, 0.6) ${ }^{1}$. Ähnlich hohe Prävalenzraten in den beiden Stichproben liegen in Bezug auf die substanzbezogenen Störungen exklusive Nikotin vor (OR: 0.9, 0.7, 0.7). Deutlich höhere Prävalenzen in der kardiovaskulären Stichprobe finden sich in den Diagnosekategorien substanzbezogene Störungen inklusive Nikotin (OR: 0.9, 1.7, 1.8*), den affektiven Störungen (OR: 1.4, 1.8*, 1.5) und den Angststörungen (OR: 1.4, $\left.1.7,2.5^{* *}\right)$. Durch die im Vergleich zur muskuloskelettalen Stichprobe niedrigere Patientenanzahl in der kardiovaskulären Stichprobe bedarf es eines höheren Prävalenzunterschieds im Vergleich zur BGS-Stichprobe, um ein signifikantes Ergebnis zu ermitteln.

\section{Diskussion}

Die vorgestellte Studie umfasst den ersten Vergleich von Prävalenzraten psychischer Störungen (4 Wochen, 12 Monate und Lebenszeit) bei Patienten mit muskuloskelettalen und kardiovaskulären Erkrankungen und Probanden einer repräsentativen Stichprobe aus der Allgemeinbevölkerung, die unter Kontrolle der Geschlechts- und Alterseffekte ermittelt wurden, wodurch der Einfluss zweier wesentlicher Moderatorvariablen auf die Prävalenz psychischer Störungen berücksichtigt wird. Durch einen Vergleich der beiden klinischen Stichproben mit den soziodemographischen Daten aus der VDR-Statistik Rehabilitation (VDR, 2000) konnte eine hohe Repräsentativität der Stichproben in Bezug auf die Gesamtheit der Rehabilitationspatienten mit diesen Erkrankungsbildern aufgezeigt werden (Härter, Baumeister, Reuter, Wunsch \& Bengel, 2002). Im Vergleich zur Gesamtheit aller Personen mit einer der beiden Indikationen ist die Repräsentativität hingegen insoweit eingeschränkt, als dass es sich bei den verwendeten Stichproben um stationär behandelte Patienten handelt, deren durchschnittlicher Schweregrad der Erkrankung und dem damit verbundenem Leidensdruck vermutlich über dem der Gesamtpopulation liegt, was auch eine höhere Prävalenz psychischer Störungen vermuten lässt.

Sowohl über die gesamte Lebenszeit als auch im 12-Monats-Zeitfenster weisen Patienten mit einer muskuloskelettalen und einer kardiovaskulären Erkrankung mit Odds Ratios von 1.4 bis 1.6 eine erhöhte Prävalenz psychischer Störungen im Vergleich zur Allgemeinbevölkerung auf. Diese Ergebnisse sind konsistent zu Wells et al. (1988), die bei Patienten mit einer chronischen Erkrankung im Vergleich zu einer Stichprobe ohne (die acht untersuchten) chronische Erkrankungen ein um $41 \%$ erhöhtes relatives Risiko für eine psychische Störung fanden.

Die Prävalenzunterschiede sind dabei auf die über alle drei Zeitfenster hinweg deutlich höheren Prävalenzen in den beiden Diagnosekategorien affektive Störungen und Angststörungen zurückzuführen (OR: 1.3-2.5). Aufgrund der im M-CIDI nicht ausreichend zu klärenden Ätiologie wurden die organisch bedingten affektiven und Angststö-

\footnotetext{
${ }^{1}$ Die fehlende Angabe in der Kategorie sonstige Störungen (4 Wochen) beruht darauf, dass es in dieser Kategorie in der kardiovaskulären Stichprobe keinen diagnostizierten Fall gibt.
} 
rungen unter die entsprechenden psychischen Störungen subsumiert. Im Hinblick auf die therapeutischen Konsequenzen gilt es hingegen diesen differenzialdiagnostisch bedeutsamen Aspekt vertieft zu betrachten. Mit einer Lebenszeit-Prävalenz für Angststörungen von $33.8 \%$ und $36.0 \%$ sind bei muskuloskelettal und kardiovaskulär erkrankten Patienten sowohl die Unterschiede zur Allgemeinbevölkerung mit $18.4 \%$, als auch die Prävalenzraten im Vergleich $\mathrm{zu}$ den weiteren psychischen Störungen am höchsten. Ein vergleichbares Bild zeigt sich bei den affektiven Störungen $(34.9 \%, 30.1 \%, 22.5 \%)$. Im Gegensatz hierzu ermittelten Wells et al. (1989) weit niedrigere Prävalenzraten affektiver Störungen und Angststörungen, bei einem allerdings ähnlich hohen Unterschied zwischen den Gruppen aktuell chronisch kranker Personen und der nicht chronisch kranken Kontrollgruppe. Für die untersuchten Personen mit muskuloskelettalen Erkrankungen (Arthritiden) ergab sich z. B. eine Lebenszeit-Prävalenz für eine Angststörung von $20.7 \%$ und für Patienten mit einer kardiovaskulären Erkrankung von $16.1 \%$ bei Hypertonie, sowie von $28.3 \%$ bei koronarer Herzerkrankung. Die Prävalenzrate für die Gruppe ohne chronische somatische Erkrankungen liegt bei $12.4 \%$.

Neben der unterschiedlichen Stichprobenzusammensetzung (verschiedene Indikationsbereiche, Altersunterschiede der Stichproben, unterschiedlicher Schweregrad der chronischen Erkrankung bzw. unterschiedlicher Gesundheitszustand der Kontrollgruppe) dürften die höheren Prävalenzen in unserer Studie auf die verschiedenen Interview-Verfahren und unterschiedliche diagnostische Kriterien zurückzuführen sein. Während unsere Ergebnisse auf dem M-CIDI und somit auf den Kriterien der aktuellen Klassifikationssysteme DSM-IV und ICD-10 beruhen, wurde in der ECA-Studie (Robins \& Regier, 1991; Wells et al., 1988) noch das Diagnostic Interview Schedule (DIS) zur Erfassung psychischer Störungen nach DSM-III verwandt, welches insbesondere bei den Angststörungen aufgrund der klinischen Bedeutsamkeitsprüfung (mit einem Arzt oder anderen Fachmann über das Symptom gesprochen? Mehr als einmal Medizin deswegen eingenommen? Schränkt es das Leben bzw. die Aktivitäten sehr ein? ) eher Prävalenzen behandlungsbedürftiger Störungen anstatt kriteriengeleiteter psychischer Diagnosen erfasst (Narrow, Rae, Robins \& Regier, 2002; Wakefield, 2002). In einer eigenen Analyse der Frage nach der Behandlungsbedürftigkeit der ermittelten (DSM-IV/ ICD-10) Störungen zeigte sich, dass insbesondere die Angsterkrankungen (v.a. die leichten spezifischen Phobien) von den Patienten teilweise als wenig beeinträchtigend erlebt werden, so dass sich die Prävalenzen der Angsterkrankungen um ca. 40\% reduzieren, während es andererseits viele Patienten gibt, die unterschwellige Störungen (z.B. Minor Depression) aufweisen, die aufgrund ihrer Symptomatik als so stark beeinträchtigend erlebt werden, dass sie behandlungsbedürftig sind (Härter \& Bengel, 2001; Härter, Reuter, Weisser et al., 2002).

Ein bemerkenswerter Unterschied zwischen den beiden klinischen Stichproben besteht bezüglich der somatoformen Störungen. Während die Prävalenzunterschiede in allen drei Zeitfenstern zwischen den Patienten mit muskuloskelettaler Erkrankung und den Probanden aus der Allgemeinbevölkerung gering sind, weisen die Patienten mit einer kardiovaskulären Erkrankung im Vergleich zur Allgemeinbevölkerung (und somit auch im Vergleich zur muskuloskelettalen Stichprobe) eine deutlich niedrigere Prävalenz somatoformer Störungen auf (OR: 0.3-0.6). Der hohe Anteil von somatoformen Störungen in der Allgemeinbevölkerung geht dabei auf die im Vergleich zu den klinischen Stichproben häufigeren spezifischen Diagnosen „Somatisierungsstörung“ und ,undifferenzierte Somatisierungsstörung " zurück (Odds Ratios der beiden klinischen Stichproben über alle Zeitfenster von 0.1-0.4), während der Unterschied zwischen den klinischen Stichproben in erster Linie durch eine höhere Prävalenz somatoformer Schmerzstörungen bei den Patienten mit einer muskuloskelettalen Erkrankung begründet ist (Härter, Baumeister, Höfler, Bengel \& Hahn, 2002). Ein Grund für den im Vergleich zu den klinischen Stichproben unerwartet hohen Anteil somatoformer Störungen in der Allgemeinbevölkerung könnte in den DSM-IV-Diagnosekriterien für eine Somatisierungsstörung liegen (American Psychiatric Association, 1994), nach denen eine Störung nur vergeben wird, falls die Symptome nicht vollständig durch einen bekannten medizinischen Krankheitsfaktor erklärt werden können. Da eine medizinische Begründung der Symptome bei Patienten mit einer chronischen Erkrankung häufig näher liegt und daher auch von den Patienten im Vergleich zu Probanden aus der Allgemeinbevölkerung eher als Grund genannt werden dürfte, könnte die höhere Prävalenz in der Allgemeinbevölkerung hierdurch erklärt werden. Neben dem vermutlich tatsächlich häufigeren Auftreten von Schmerzstörungen bei Patienten mit muskuloskelettalen Erkrankungen (unspezifische Rückenschmerzen etc.), könnte ein unterschiedlicher Attributionsstil der Patienten bei der Erklärung der somatischen Symptome auch im Vergleich der beiden klinischen Stichproben den Unterschied der Prävalenzraten bewirken. Bislang bietet das M-CIDI allerdings noch keine Möglichkeit, derartige Einflussfaktoren auf die Diagnosestellung zu kontrollieren.

Als vierte bedeutsame Kategorie psychischer Störungen werden Prävalenzen substanzbezogener Störungen berichtet, sowohl exklusive als auch inklusive Nikotinabhängigkeit. Die getrennte Betrachtung der Nikotinabhängigkeit und die Berechnung der Prävalenz psychischer Störungen insgesamt ohne Nikotinabhängigkeit erfolgte dabei aufgrund des hohen Anteils nikotinabhängiger Probanden in den Stichproben. Durch dieses Vorgehen soll eine Überschätzung der Prävalenz psychischer Störungen vermieden werden, insbesondere da Referenzstudien wie Wells et al. (1988) und die großen epidemiologischen Bevölkerungssurveys (Kessler et al., 1994; Robins \& Regier, 1991) keine Prävalenzen zur Nikotinabhängigkeit berichten. Auf der anderen Seite unterstreicht die deutlich erhöhte Prävalenz substanzbezogener Störungen inklusive Nikotin der klinischen Stichproben im Vergleich zur Allgemeinbevölkerung die Bedeutung des Rauchens als einer der wesentlichen assoziierten Faktoren bei chronischen Erkrankungen. 
Relativ niedrig fällt die Prävalenz substanzbezogener Störungen ohne Nikotin aus. Mit einer Lebenszeitprävalenz von $8.4 \%$ und $6.4 \%$ bei den muskuloskelettal und kardiovaskulär erkrankten Patienten, sowie $8.6 \%$ in der Allgemeinbevölkerung liegen die Prävalenzen deutlich unter den Raten von Wells et al. (1989), die für die drei entsprechenden Stichproben chronisch erkrankter Probanden (s. o.) Prävalenzen von $21.9 \%$ bis $33.4 \%$ ermittelten, sowie 19.6\% für die Stichprobe nicht chronisch kranker Probanden. Ein Hauptgrund für diesen großen Unterschied dürfte in den unterschiedlichen AuswertungsAlgorithmen für die Diagnose Alkoholmissbrauch liegen. In einer Berechnung dieser Diagnose ohne gleichzeitige Abhängigkeit entsprechend der weniger strengen Kriterien des schädlichen Gebrauchs nach ICD-10 ergeben sich Prävalenzsteigerungen in der BGS-Stichprobe von $2.2 \%$ (DSM-IV) auf 9.8\% (ICD-10) bzw. von 9.9\% für irgendeine substanzbezogene Störung ohne Nikotin auf 16.7\%. Diese Prävalenzraten nach ICD-10-Kriterien liegen allerdings immer noch deutlich unter den Prävalenzraten amerikanischer Studien, die im internationalen Vergleich von Bevölkerungssurveys stets mit die höchste Prävalenz substanzbezogener Störungen aufweisen (Andrews, Henderson \& Hall, 2001; Kessler et al., 1994; Robins \& Regier, 1991; Wittchen, 2000; WHO International Consortium in Psychiatric Epidemiology, 2000). Ein Teil der niedrigeren Prävalenz in unserer Studie im Vergleich zu Wells et al. (1989) dürfte somit auch auf kulturspezifische Eigenheiten, wie z. B. ein unterschiedlicher Substanzkonsum, unterschiedliche Folgen aus dem Konsum, eine unterschiedliche Bewertung des Konsums oder ein unterschiedliches Maß an sozialer Erwünschtheit beim Beantworten der Interview-Fragen zurückgehen.

Die Ergebnisse legen nahe, dass bei der Rehabilitation von Patienten mit einer chronischen Erkrankung verstärkt auf die Diagnostik und Behandlung komorbider psychischer Störungen geachtet werden sollte. Obwohl es sich bei der hier gewählten Vergleichsgruppe nicht um eine gesunde Kontrollgruppe handelt, sondern um die (teilweise ebenfalls beeinträchtigte) Allgemeinbevölkerung, ergeben sich für die klinischen Stichproben deutlich erhöhte Prävalenzraten psychischer Störungen, die zudem in Verbindung mit einer chronischen Erkrankung einen negativen, wahrscheinlich kumulativen Effekt auf die Lebensqualität, die Mortalität, die Compliance und die Behandlung der Patienten haben (Candilis et al., 1999; Härter, Reuter, Weisser et al. 2002; Satariano \& Ragland, 1994; Sherbourne, Wells, Meredith, Jackson \& Camp, 1996). Entsprechend den Ergebnissen gilt es hierbei im Besonderen auf komorbid vorliegende Angst- und affektive Störungen zu achten. Zur einfachen und validen Erfassung können Screeningverfahren wie z.B. die HADS (Herrmann, Buss \& Snaith, 1995) eingesetzt werden, die hinreichend sensitiv und spezifisch psychische Störungen erkennen helfen (Härter, Reuter, Groß-Hardt \& Bengel, 2001; Reuter \& Härter, 2001). Werden komorbide Störungen diagnostiziert, gilt es eine vertiefende Diagnostik an das Screening anzuschließen und eine entsprechende Behandlung in die Wege zu leiten, um der Beeinträchtigung durch die psychische Störung entgegen zu wirken und eine weitere Chronifizierung der körperlichen Erkrankung, sowie ein steigendes Mortalitätsrisiko zu verhindern (Härter, 2002; Härter et al., 2002; Heßlinger et al., 2002; Linton, 2000).

\section{Förderung und Danksagung}

Diese Arbeit entstand im Rahmen des Forschungsprojektes „Epidemiologie psychischer Störungen in der medizinischen Rehabilitation“"(01 GD 9802/4 und 01 GD 0101) des Rehabilitationswissenschaftlichen Forschungsverbundes Freiburg/Bad Säckingen. Das Projekt wird vom Bundesministerium für Bildung und Forschung (BMBF) und vom Verband Deutscher Rentenversicherungsträger (VDR) gefördert. Der Zusatzsurvey „Psychische Störungen“" des Bundesgesundheitssurveys 98 (01 EH970/8) wurde vom BMBF gefördert. Wir danken dem RobertKoch-Institut für die Bereitstellung des BGS-Kernsurveys (Public Use File BGS98, 2000). Weiter danken wir allen Rehabilitationskliniken, durch deren Kooperation diese Studie erst ermöglicht wurde.

\section{Literatur}

American Psychiatric Association (1994). Diagnostic and Statistic Manual of Mental Disorders. $4^{\text {th }}$ ed. Washingtion DC: American Psychiatric Press.

Bellach, B. M. (1999). Editorial: Der Bundesgesundheitssurvey 1998 - Erfahrungen, Ergebnisse, Perspektiven [Sonderheft]. Gesundheitswesen, 61, 55-56.

Candilis, P. J., McLean, R. Y., Otto, M. W., Manfro, G. G., Worthington, J. J., III, Penava, S. J., Marzol, P. C. \& Pollack, M. H. (1999). Quality of life in patients with panic disorder. Journal of Nervous Mental Disease, 187, 429434.

Chignon, J. M., Lepine, J. P. \& Ades, J. (1993). Panic disorder in cardiac outpatients. American Journal of Psychiatry, 150, 780-785.

Cohen-Cole, S. A., Brown, F. W. \& McDaniel, J. S. (1993). Assessment of depression and grief reactions in the medically ill. In A. Stoudemire \& B. S. Fogel (Eds.), Psychiatric Care of the Medical Patient (pp. 53-69). New York: Oxford University Press.

Frank, R. G., Beck, N. C., Parker, J. C., Kashani, J. H., Elliot, T. R., Haut, A. E., Smith, E., Atwood, C., Brownlee-Duffect, M. \& Kay, D. R. (1988). Depression in rheumatoid arthritis. Journal of Rheumatology, 15, 920-925.

Frasure-Smith, N., Lespérance, F. \& Talajic, M. (1993). Depression following myocardial infarction: impact on 6-month survival. JAMA, 270, 1819-1861.

Goldberg, D. \& Williams, P. (1988). A user's guide to the General Health Questionnaire. Windsor: NFER-Nelson.

Gonzalez, M. B., Snyderman, T. B., Colket, J. T., Arias, R. M., Jiang, J. W., O'Connor, C. M. \& Krishman, K. R. (1996). Depression in patients with coronary artery disease. Depression, 4, 57-62.

Härter, M. (2002). Ätiologie psychischer Störungen bei chronischen körperlichen Erkrankungen. Die Rehabilitation, 41, 357-366.

Härter, M., Baumeister, H., Reuter, K., Wunsch, A. \& Bengel, J. (2002). Epidemiologie komorbider psychischer Störungen bei Rehabilitanden mit muskuloskelettalen und kardiovaskulären Erkrankungen. Die Rehabilitation, 41, 367-374. 
Härter, M., Baumeister, H., Höfler, M., Bengel, J. \& Hahn, D. (2002). Somatoform and mental disorders in somatically ill patients with musculoskeletal and cardiovascular diseases. Poster presented at the International Congress on „Somatoform Disorders - New Approaches to Classification and Treatment", Marburg.

Härter, M. \& Bengel, J. (2001). Epidemiologie psychischer Störungen in der medizinischen Rehabilitation. Unveröffentlichter Abschlussbericht (01 GD 9802/4), Universität Freiburg.

Härter, M., Reuter, K., Aschenbrenner, A., Schretzmann, B., Marschner, N., Hasenburg, A. \& Weis, J. (2001). Psychiatric disorders and associated factors in cancer: Results of an interview study with patients in inpatient, rehabilitation and outpatient treatment. European Journal of Cancer, 37, $1385-1393$.

Härter, M., Reuter, K., Gross-Hardt, K. \& Bengel, J. (2001). Screening for anxiety, depressive and somatoform disorders in rehabilitation - validity of HADS and GHQ-12 in patients with musculoskeletal disease. Disability and Rehabilitation, 23, 737-744.

Härter, M., Reuter, K., Weisser, B., Schretzmann, B., Aschenbrenner, A. \& Bengel, J. (2002). A descriptive study of psychiatric disorders and psychosocial burden in rehabilitation patients with musculoskeletal diseases. Archives of Physical Medicine and Rehabilitation, 83, 461-468.

Herrmann, C., Buss, U. \& Snaith, R. P. (1995). Hospital Anxiety and Depression Scale - Deutsche Version (HADS-D). Bern: Huber.

Heßlinger, B., Härter, M., Barth, J., Klecha, D., Bode, C., Walden, J., Bengel, J. \& Berger, M. (2002). Komorbidität von depressiven Störungen und kardiovaskulären Erkrankungen - Implikationen für Diagnostik, Pharmako- und Psychotherapie. Der Nervenarzt, 73, 205-218.

Jacobi, F., Wittchen, H.-U., Müller, N., Hölting, C., Sommer, S., Lieb, R., Höfler, M. \& Pfister, H. (2002). Estimating the prevalence of mental and somatic disorders in the community: Aims and methods of the German National Health Interview and Examination Survey. International Journal of Methods in Psychiatric Research, 11, 1-18.

Kessler, R. C., McGonagle, K. A., Zhao, S., Nelson, C. B., Hughes, M., Eshleman, S., Wittchen, H.-U. \& Kendler, K. S. (1994). Lifetime and 12-Month Prevalence of DSM-IIIR Psychiatric Disorders in the United States. Archives of General Psychiatry, 51, 8-19.

Linton, S. J. (2000). A review of psychological risk factors in back and neck pain. Spine, 25, 1148-1156.

Magni, G., Marchetti, M., Moreschi, C., Merskey, H. \& Lucchini, S. R. (1993). Chronic musculoskeletal pain and depressive symptoms in the national health and nutrition examination. I. Epidemiologic follow-up study. Pain, 53, 163-168.

Narrow, W. E., Rae, D. S., Robins, L. N. \& Regier, D. A. (2002). Revised prevalence estimates of mental disorders in the United States. Using a clinical significance criterion to reconcile 2 surveys' estimates. Archives of General Psychiatry, 59, 115-123.

Penninx, B. W. J. H., Beekman, A. T. F., Honig, A., Deeg, D. J. H., Schoevers, R. A., van Eijk, J. T. E. \& van Tilburg, W. (2001). Depression and cardiac mortality. Results from a community-based longitudinal study. Archives of General Psychiatry, 58, 221-227.

Polatin, P. B., Kinney, R. K., Gatchel, R. J., Lillo, E. \& Mayer, T. G. (1993). Psychiatric illness and chronic low-back pain. The mind and the spine - which goes first? Spine, 18, 6671.

Pratt, L. A., Ford, D. E., Crum, R. M., Armenian, H. K., Gallo, J. \& Eaton, W. W. (1996). Depression, psychotropic medication, and risk of myocardial infarction. Circulation, 94, 3123-3129.

Public Use File BGS98 (2000). Bundes-Gesundheitssurvey 1998. Berlin, Robert Koch-Institut.
Reuter, K. \& Härter, M. (2001). Screening for mental disorders in cancer patients - discriminant validity of HADS and GHQ-12 assessed by standardized clinical interview. International Journal of Methods in Psychiatric Research, 10, 86-96.

Robins, L. N. \& Regier, D. A. (1991). Psychiatric disorders in America. The Epidemiological Catchment Area Study. New York: Free Press.

Satariano, W. A. \& Ragland, D. R. (1994). The effect of comorbidity on 3-year survival of women with primary breast cancer. Annals of Internal Medicine, 120, 104-110.

Sherbourne, C. D., Wells, K., Meredith, L. S., Jackson, C. A. \& Camp, P. (1996). Comorbid anxiety disorder and the functioning and well-being of chronically ill patients of general medical providers. Archives of General Psychiatry, $53,889-895$.

StataCorp. (2001). Stata Statistical Software, release 7.0. College Station, TX: Stata Corporation.

VDR (2000). VDR Statistik Rehabilitation. Leistungen zur Rehabilitation und zusätzliche Leistungen der gesetzlichen Rentenversicherung im Jahr 1999. Frankfurt: Verband Deutscher Rentenversicherungsträger.

Wakefield, J. C. (2002). Lowered estimates - but of what? Archives of General Psychiatry, 59, 129-130.

Wells, K. B., Golding, J. M. \& Burnam M. A. (1988). Psychiatric disorder in a sample of the general population with and without chronic medical conditions. American Journal of Psychiatry, 145, 976-981.

Wells, K. B., Golding, J. M. \& Burnam M. A. (1989). Affective, substance use, and anxiety disorders in persons with arthritis, diabetes, heart disease, high blood pressure, or chronic lung conditions. General Hospital Psychiatry, 11, 320-327.

Wittchen, H.-U. (2000). Schlussbericht Zusatzsurvey, „Psychische Störungen “ (Bundesgesundheitssurvey '98): Häufigkeit, psychosoziale Beeinträchtigungen und Zusammenhänge mit körperlichen Erkrankungen. Bonn: Bundesministerium für Bildung und Forschung.

Wittchen, H.-U. \& Pfister, H. (1997). Instruktionsmanual zur Durchführung von DIA-X-Interviews. Swets Test Services, Frankfurt.

Wittchen, H.-U., Robins, L. N., Cottler, L. B., Sartorius, N., Burke, J. D., Regier, D. \& participants of the WHO/ ADAMHA field trials. (1991). Cross-cultural feasibility, reliability and sources of variance of the Composite International Diagnostic Interview (CIDI) - Results of the multicenter WHO/ADAMHA field trials (Wave I). British Journal of Psychiatry, 159, 645-653.

Wittchen, H.-U., Weigel, A. \& Pfister, H. (1996). DIA-X-Diagnostisches Expertensystem. Frankfurt: Swets Test Services.

World Health Organization (WHO). (1990). Composite International Diagnostic Interview (CIDI, Version 1.0). Geneva: World Health Organization.

World Health Organization (WHO). (1997). Composite International Diagnostic Interview (CIDI, Version 2.1). Geneva: World Health Organization.

Manuskript eingereicht: $\quad 30.07 .2002$ Manuskript angenommen: 12. 11. 2002

Harald Baumeister, Dipl.-Psych.

PD Dr. Dr. Martin Härter, Dipl.-Psych.

Abteilung für Psychiatrie und Psychotherapie

Universitätsklinik Freiburg

Hauptstraße 5

79104 Freiberg

E-Mail: harald_baumeister@psyallg.ukl.uni-freiburg.de

E-Mail:martin_haerter@psyallg.ukl.uni-freiburg.de 